\section{The importance for immunoregulation for long-term cancer control}

\author{
Alberto Fusi ${ }^{1,2}$ \& Angus Dalgleish ${ }^{*, 1}$
}

Immune checkpoint blockades have recently emerged as a breakthrough treatment for solid tumors showing high response rates and long durability. In melanoma, the combination of ipilimumab with nivolumab showed high efficacy. However, still half the patients do not respond to this treatment. In order to increase the therapeutic ratio in melanoma and other cancers, different approaches are under evaluation. Three relevant questions are at the moment driving the research community: how to maximize benefit while minimizing toxicity; how to better identify patients who are more likely to benefit from immunotherapy; how to convert nonresponders into responders. In this review we summarize the most recent findings and we outline the most likely future challenges.

First draft submitted: 21 February 2017; Accepted for publication: 24 April 2017; Published online: 4 August 2017

In 2016, cancer immunotherapy was named 'Advance of the Year' by the American Society of Clinical Oncology, and it has in fact emerged as a breakthrough treatment for cancers like melanoma, known to be resistant to classic therapeutic approaches such as chemotherapy. A number of cytokines, including IL-2, IFN- $a$ and IL-12, and vaccines have been tried with various degrees of success with a survival benefit being observed in a very small fraction of patients [1]. Anti-CTLA-4 and anti-PD1 antibodies have extended the survival benefit to a much larger number of patients, making the checkpoint blockades the most successful drugs of the past few years.

Of all cancer types, melanoma has been the most investigated. More immunotherapy drugs are indeed approved for melanoma than for any other type of cancer and more are in development. Melanoma has always shown higher responsiveness to immunotherapeutic approaches compared with the other solid tumors but, despite the recent very significant improvements, more than half the patients still do not respond to treatment. In order to increase efficacy of the immunotherapy in melanoma and other cancers, different approaches are under evaluation. There is a rationale and, therefore, a potential role for combination treatments of immunotherapy with chemotherapy, radiotherapy or further immunotherapy. But the increased potential benefit of a combination treatment may come at a price of a less favorable toxicity profile. Two relevant questions are at the moment driving the research of the clinical and scientific community focused on immunotherapeutic treatment strategies: how to maximize benefit while minimizing toxicity; how to better identify patients who are more likely to benefit from immunotherapy; how to convert nonresponders into responders.

\section{Checkpoint blockades: state of art}

Ipilimumab is a fully humanized anti-CTLA- 4 antibody, which enables cytotoxic T-cell activity and perpetuates immune responses by blocking the CTLA- 4 receptor. Ipilimumab was the first drug

'Infection \& Immunity Research Center - St George's, University of London, Cranmer Terrace, London, SW17 ORE, UK

${ }^{2}$ Charité Comprehensive Cancer Center - Charité-Invalidenstr. 80, 10115 Berlin, Germany

*Author for correspondence: dalgleis@sgul.ac.uk

\section{KEYWORDS}

- atezolizumab - biomarkers

- checkpoint inhibitors

- immunotherapy

- ipilimumab • melanoma

- nivolumab • NSCLC

- pembrolizumab 
to show a survival advantage in metastatic melanoma $[2,3]$. Despite the low rate of response, a recent pooled analysis showed that around 20\% could be considered long-term survivors, being alive at 5 years after treatment [4].

Anti-PD1 and anti-PDL1 antibodies have more recently emerged as breakthrough drugs for melanoma, showing high response rates and long durability. In the CheckMate 066 study [5] comparing the anti-PD1 antibody nivolumab with dacarbazine in patients with BRAF wildtype, unresectable, advanced or metastatic melanoma previously untreated, an overall response rate (ORR) of $40 \%$ was observed for nivolumab compared with $14 \%$ for dacarbazine. Overall survival (OS) was significantly increased in those treated with nivolumab (1 year survival rate 73 vs $42 \%$ ) and progression-free survival (PFS) was also increased with nivolumab (median 5.1 vs 2.2 months). Similar results were observed in the CheckMate 037 Phase III trial [6] in 405 patients previously treated with ipilimumab who were randomly assigned in a $2: 1$ ratio to either nivolumab or chemotherapy (ORR 38 vs $10 \%$ respectively). Patients on nivolumab had a longer median duration of response (median not reached vs 3.5 months for chemotherapy treated patients). In the large CheckMate 067 [7], 945 previously untreated patients with unresectable stage III or IV melanoma were randomized in a 1:1:1 ratio to nivolumab alone, nivolumab plus ipilimumab or ipilimumab alone. The median PFS with both the combination and nivolumab alone was superior to ipilimumab alone (11.5 vs 2.9 months and 6.9 vs 2.9 months) with an ORR for the combination, nivolumab alone and ipilimumab alone of 58 , 44 and 19\%, respectively. OS data have been recently presented at the American Association for Cancer Research (AACR) 2017 meeting. The 2 -year OS rate was $64 \%$ for patients randomly assigned to nivolumab plus ipilimumab and 59 and $45 \%$ for patients treated with single agent nivolumab or ipilimumab, respectively. The combination showed a decrease in the risk of death of the $12 \%$ compared with single agent nivolumab (descriptive analysis) [8].

The anti-PD1 antibody pembrolizumab was granted approval for the treatment of patients with metastatic melanoma based on the results of a very large Phase I study (KEYNOTE-001). In the pooled analysis of 655 patients with melanoma, an ORR of $34 \%$ was observed with a $6 \%$ complete response rate. Median PFS was 5.2 months with an OS rate at 2 years of $50 \%$ [9]. In the KEYNOTE-006, 834 patients with advanced melanoma were randomized to receive pembrolizumab $10 \mathrm{mg} / \mathrm{kg}$ every 2 or 3 weeks versus ipilimumab. The response rate was improved with pembrolizumab every 2 weeks $(33.7 \%)$ and every 3 weeks $(32.9 \%)$, as compared with ipilimumab (11.9\%). PFS rate at 6 months was significantly higher for patients receiving pembrolizumab $(47.3 \%$ for pembrolizumab every 2 weeks and $46.4 \%$ for pembrolizumab every 3 weeks) in comparison with ipilimumab (26.5\%). Similarly, OS was improved with an estimated OS rate at 12 months of $74.1,68.4$ and $58.2 \%$ for pembrolizumab every 2 weeks, pembrolizumab every 3 weeks and ipilimumab, respectively [10].

In March 2015, nivolumab received accelerated approval by the US FDA as secondline treatment for metastatic non-small-cell lung cancer (NSCLC) of squamous histology that has progressed on or after platinum-containing chemotherapy, based on the results of the CheckMate 017 study [11]. In the study, nivolumab showed a statistically significant prolongation of the median OS by 3.2 months ( 9.2 vs 6.0 months) compared with single agent docetaxel, with a relative $41 \%$ reduction in the risk of death. Median PFS (3.5 vs 2.8 months), ORR ( 20 vs $9 \% ; \mathrm{p}=0.008$ ) and 1 -year OS rate (42 vs $24 \%$ ) were also superior in the nivolumab arm. In October of the same year, the FDA expanded the indication for nivolumab to include patients with nonsquamous NSCLC. Approval was based on demonstration of an improvement in OS as shown in the CheckMate 057 study [12]. In this multicenter, open-label, randomized trial patients with metastatic nonsquamous NSCLC with progression on or after platinum-based chemotherapy were randomized 1:1 to receive nivolumab or docetaxel. Nivolumab demonstrated improved OS compared with docetaxel, with a median OS of 12.2 versus 9.4 months. The median duration of response was 17 months in the nivolumab arm and only 6 months in the docetaxel arm.

The other anti-PD1 antibody, pembrolizumab, was granted FDA-accelerated approval to treat patients with advanced NSCLC whose disease has progressed after other treatments and with tumors that express PD-L1 (tumor proportion score $>1 \%$ ). Approval was based on the results from the NSCLC cohorts of the large Phase I trial KEYNOTE-001 [13]. In the 
overall population of 495 patients with advanced NSCLC, the ORR, PFS and OS were $19.4 \%$, 3.7 and 12 months, respectively. Patients with a PD-L1 score higher than 50\% showed significantly greater benefit. Results have been recently confirmed and extended to first-line setting by the KEYNOTE-024 trial [14]. In this Phase III study, 305 patients with previously untreated NSCLC and a PD-L1 expression on at least 50\% of tumor cells were randomized to receive either pembrolizumab or the investigator's choice of platinum-based chemotherapy. Median PFS was 10.3 month in the pembrolizumab group with a median duration of response not reached versus 6.0 months in the chemotherapy group with a median duration of response of 6.3 months. Pembrolizumab approval was consequently extended to first-line setting for patients with a tumor proportion score $>50 \%$.

Atezolizumab was the first ever anti-PD-L1 antibody approved by FDA. It is indicated for patients with metastatic NSCLC whose disease progressed during or following platinumcontaining chemotherapy and for patients with urothelial carcinoma. Approval for lung cancer was based on the results of a Phase III study, which showed improved OS and PFS for patients with NSCLC previously treated with atezolizumab in comparison to docetaxel (median OS of 13.8 vs 9.6 months) [15]. Atezolizumab showed efficacy also in patients with urothelial carcinoma previously treated with a platinum-based regimen. The reported ORR was 16\% for all the patients and 28\% for those with PD-L1 expression $>5 \%$ [16]. Results are in keeping with the ORR observed in early phase studies with the PD-1 antibodies nivolumab [17] and pembrolizumab [18]. More recently, the other anti-PD-L1 antibody durvalumab has granted priority review status by the FDA for the treatment of patients with locally advanced or metastatic urothelial carcinoma whose disease has progressed during or after one standard platinum-based regimen based on the results of a Phase I/II study. Sixty-one patients were treated with durvalumab $10 \mathrm{mg} / \mathrm{kg}$ every 2 weeks. The ORR was $31 \%$ in the 42 evaluable patients with responses ongoing in 12 of the 13 responding patients at a median follow-up of 6.5 months. ORR increased up to 46.4\% in PD-L1-positive patients [19].

Significant efficacy and prolongation of PFS and OS have also been observed for nivolumab in patients with metastatic renal cancer, classic Hodgkin lymphoma and advanced head and neck cancer. In patients with metastatic renal cancer pretreated with antiangiogenic therapy, nivolumab showed superiority compared with everolimus in terms of OS (25.0 vs 19.6 months) and ORR (25 vs 5\%) [20]. Higher ORR and prolonged OS were also observed in the difficult setting of patients with recurrent squamous cell carcinoma of the head and neck (SCCHN) whose disease had progressed within 6 months after platinum-based chemotherapy. Patients were randomized to receive nivolumab versus standard single-agent systemic therapy (methotrexate, docetaxel or cetuximab). The median OS was 7.5 months in the nivolumab group versus 5.1 months in the group that received standard therapy and the ORR 13.3 and 5.8\%, respectively [21]. Nivolumab has received FDA approval for the treatment of renal cancer patients who have received prior antiangiogenic therapy and for the treatment of patients with recurrent or metastatic SCCHN with disease progression on or after platinum-based therapy.

In May 2016, nivolumab was granted accelerated approval for the treatment of patients with classical Hodgkin lymphoma that has relapsed or progressed after autologous hematopoietic stem cell transplantation and post-transplantation brentuximab vedotin based on the results of a Phase II study. At a median follow-up of 8.9 months, an ORR of the $66.3 \%$ was observed among 80 patients treated with a median duration of response of around 9 months [22]. More recently, also pembrolizumab granted FDA approval for the treatment of patients with refractory classical Hodgkin lymphoma, or those who have relapsed after three or more prior lines of therapy based on the results of the Phase II KEYNOTE-087 trial which showed an ORR of $69 \%$ with an estimated median response duration of 11.1 months [23].

\section{Predictive biomarkers, more than just PD-1/PDL-1}

The identification of patients potentially responsive to immunotherapy is one of the challenges clinicians are facing at the moment. Biomarkers can help clinicians to select patients for immunotherapy, avoiding unwanted side effects for those patients who are not likely to benefit from immunotherapy.

The PD-1/PD-L1/PD-L2 pathway is a key immune checkpoint. PD-1 is a transmembrane glycoprotein, which acts as a T-cell coinhibitory receptor. After cognate antigen detection, 
PD-1 is rapidly upregulated on activated $\mathrm{T}$ cells, while its expression decreases after antigen clearance. Binding of PD-1 by its ligand PD-L1 results in inhibition of the activated T cells, switching off the T-cell response. PD-L1 is constitutively expressed on T- and B-cells as well as on macrophages and is also expressed in parenchymal tissues such as endothelial and muscle cells. The expression of PD-L1 is greatly increased following stimulation by inflammatory cytokines. Given that both PD-1 and PD-L1 are upregulated in tissues during inflammation, and that their interaction determines functional inhibition of lymphocytes, PD-1/PD-L1 plays a key role in the local inflammatory response and prevention of immune-mediated damage. In patients with cancer, because of the constitutive antigen exposure, PD-1 is constantly expressed on effector $\mathrm{T}$ cells and has become a marker of unresponsiveness or exhaustion of the T cell. PD-L1 can be expressed on cancer cells and on cells of the tumor microenvironment. Blocking the PD-1/PD-L1 pathway can, therefore, potentially restore the effector function of tumor-specific $\mathrm{T}$ cells.

The question of whether expression of PD-L1 correlates with treatment outcomes with antiPD-1 and anti-PD-L1 monoclonal antibodies has been addressed in most of the pivotal trials.

PD-L1 expression has been generally assessed on tumor specimens by immunohistochemistry (IHC). A correlation between expression of PD-L1 on tumor cells and response to treatment was first seen in a Phase I study of nivolumab in solid tumors [5]. PD-L1-positive status was defined as $\geq 5 \%$ cell membrane staining of any intensity, evaluated through IHC using the murine antihuman PD-L1 monoclonal antibody $5 \mathrm{H} 1$. Multiple tumor slides were tested for each patient, and a patient was considered to have a PD-L1-positive tumor if one biopsy sample was positive. Twenty-five patients were categorized as PD-L1 positive and 21 as negative. A treatment response was seen in nine PD-L1-positive patients (36\%) whereas none of the PD-L1 negative had a response. The assessment of PD-L1 positivity was performed in an operator-dependent manner. PD-L1 expression in subsequent clinical trials of nivolumab has been assessed by means of a novel automated PD-L1 assay using a rabbit monoclonal antibody (clone 28-8).

Using this platform of detection in melanoma patients, previously treated with ipilimumab, nivolumab showed an ORR of $44 \%$ in PD-L1positive patients (defined as $\geq 5 \%$ tumor cell membrane). However, a significant proportion of patients $(20 \%)$ defined as being PD-L1 negative also had a treatment response. Similar findings were also observed in the CA209-066 trial [24] in which nivolumab was compared with dacarbazine in patients with BRAF wild-type melanoma. Nivolumab was associated with a higher ORR than dacarbazine, irrespective of PD-L1 status. In PD-L1-positive patients, the ORR was $52.7 \%$ for nivolumab-treated patients and $10.8 \%$ for those receiving dacarbazine. However, the ORR was also higher with nivolumab compared with dacarbazine in patients with negative (or indeterminate) PD-L1 status, even though the response rate was not as high and the difference not as marked (33.1 vs $15.7 \%)$. For patients treated with nivolumab, the median OS was not reached in either PD-L1 subgroup. For the patients treated with dacarbazine, the median OS was slightly greater in the patients with PD-L1-positive melanoma (12.4 vs 10.2 months), suggesting an interaction between PD-L1 expression and outcome with chemotherapy.

In the CheckMate 057 study [12] of nivolumab versus docetaxel in patients with nonsquamous NSCLC, PD-L1 expression appeared to be a useful predictive factor of response, regardless of the cut-off used (1, 5 or $10 \%)$. Conversely, in the Phase III CheckMate 017 study [11] comparing docetaxel with nivolumab in 272 previously treated patients with advanced or metastatic squamous NSCLC, the survival benefit and improved ORR of nivolumab were independent of PD-L1 expression level, regardless of the cut-off level used to define PD-L1 status ( $\geq 1,5$ or $10 \%)$.

The pivotal CheckMate 067 trial compared the combination of nivolumab plus ipilimumab with nivolumab and ipilimumab monotherapies in 945 treatment-naive patients with advanced melanoma. Patients were stratified according to PD-L1 expression, BRAF mutation and disease stage [7]. Subgroup analysis by PD-L1 status showed higher ORR for patients treated with the combination compared with nivolumab monotherapy in both PD-L1-positive patients (72.1 vs $57.5 \%$ ) and PD-L1-negative patients (54.8 vs $41.3 \%$ ), using a $5 \%$ cut-off. However, the median PFS for PD-L1-positive patients was 14 months for both combination therapy and single agent nivolumab compared with 3.9 months in the 
ipilimumab group. In PD-L1-negative patients, median PFS was higher in the combination arm compared with nivolumab monotherapy (11.2 vs 5.3 months). Of note, the fraction of patients with positive PD-L1 expression in this study was only $21.7 \%$, lower than that observed in other studies of anti-PD-1 therapies.

In the clinical trials with pembrolizumab, the $22 \mathrm{C} 3$ mouse monoclonal antibody has been used to assess PD-L1 expression. In the pivotal KEYNOTE-006 [24] Pembrolizumab (given either every 2 or every 3 weeks) was compared with ipilimumab in patients with advanced melanoma. PD-L1 status was assessed in archival or newly obtained tumor samples by means of IHC at a central laboratory before randomization and positivity was defined as membranous PD-L1 staining in at least $1 \%$ of tumor cells. Patients receiving pembrolizumab showed higher ORR and improved survival. Estimated 12-month survival rates were $74.1 \%$ for pembrolizumab every 2 weeks, $68.4 \%$ for pembrolizumab every 3 weeks and $58.2 \%$ for ipilimumab. The pembrolizumab benefit was observed across all subgroups and for the two regimens, with the exception of $18 \%$ of patients with PD-L1-negative tumors.

Taken together, these studies suggest that while PD-L1 expression has potential as predictive biomarker, it cannot be currently used for patient selection for single agent anti-PD1s as a significant number of patients classified as PD-L1 negative still benefit from treatment. In the complex network of interaction among cells of the immune system, absence of expression of PD-L1 on cancer cells or in the tumor microenvironment does not preclude that some antitumor activity can be restored by anti-PD1 antibodies via other immunological pathways.

PD-L1 could help to identify patients who can receive greater benefit for the combination of ipilimumab and nivolumab over single agent nivolumab. However, this should be done with caution as the CheckMate 067 study was not powered to answer this question, and patients with a PD-L1-positive tumor showed a significantly higher ORR which may lead to surgery, no evidence of (NED) disease in some cases and potentially long benefit.

Expression of PD-L1 alone may not be sufficient to identify patients responding to immunotherapy and the presence of activated T cells within the tumor microenvironment appears to be very important. A study of 46 patients with metastatic melanoma treated with pembrolizumab showed that presence of $\mathrm{CD} 8^{+} \mathrm{T}$ cells prior to treatment located at the invasive tumor margin and inside the tumor was associated with expression of PD-1 and PD-L1 and predicted response to therapy [25]. Similar findings with respect of the presence of tumor-infiltrating lymphocytes (TILs) were previously observed for patients treated with ipilimumab [26]. The molecular mechanisms explaining absence of a T-cell response have not been fully identified. However, recent analyses of human melanoma metastases by exome sequencing, gene expression profiling and IHC have revealed that many tumors that lack a T-cell signature showed alterations in the $\mathrm{Wnt} / \beta$-catenin signaling pathway [27].

Emerging data have suggested that other biomarkers based on immunoprofiling and mismatch repair deficiency and mutational burden may useful predictors of treatment response to anti-PD1 antibodies.

In an analysis of baseline tumor biopsy, samples from patients with melanoma enrolled in the KEYNOTE-001 study of pembrolizumab, both the IFN-a 10 gene and expanded-immune 28-gene signatures correlated with improved ORR and PFS [21]. Similarly, data from a Phase I study of patients with RCC treated with nivolumab reported that baseline upregulation of genes known to be upregulated by ipilimumab in melanoma, together with other immunorelated genes, was strongly correlated with the outcome [20]. Also, tumors with genetic defects in mismatch repair harbor many more mutations than tumors of the same type without such repair defects. A strong correlation between defects in mismatch repair and response to pembrolizumab has been reported in patients with colorectal cancer and other solid tumors, and it is becoming a major field of interest [28]. At the recent The Society for Immunotherapy of Cancer (SITC) meeting, Weber et al. presented a serum test able to identify patients with advanced melanoma who are more likely to respond to immunotherapy [29]. The potential responders were associated with complement system, acute phase and wound healing functions. The test is under further evaluation in a larger cohort of patients. Previous studies in cancer vaccine trials have indicated that nonresponders have higher levels of inflammation markers (unpublished observations). Chronic inflammation is an inhibitor of cell mediated 
immune responses [30] and using anti-inflammatory agents in murine models significantly enhances antitumor responses.

A novel immunogenomic analysis for patients with metastatic melanoma receiving single agent nivolumab has been presented at the recent AACR meeting. In the group of patients receiving single agent nivolumab, baseline inflammatory gene expression signatures correlated with response and response to treatment was also associated with increase in CD8 and natural killer (NK) cells and decreases in macrophages [31].

The tumor mutational load has been also identified as a potential marker. The genetic rearrangements of tumor cells can result in the generation of tumor-specific peptides with the potential to bind major histocompatibility complex molecules, and therefore be recognized by the immune system as neoantigens specific for the cancer cells. In a retrospective analysis of patients with different solid tumors treated with single agent checkpoint inhibitors, cancer entities with high mutational loads showed higher response rates to checkpoint blockades compared with cancer with low mutational burden. Melanoma, NSCLC and SCCHN are typically cancer with a mutational load at least a twofold higher compared with cancer entities like sarcomas or thyroid cancer [32]. However, also within single tumor entities there was a wide range of mutational burden.

In NSCLC, smokers have a higher mutational load, and they tend to respond better to anti-PD1 antibodies. In a cohort of $16+18$ NSCLC patients, higher somatic nonsynonymous mutation burden was associated to a molecular smoking signature and with clinical efficacy of pembrolizumab [33]. Another study showed that patients with metastatic urothelial carcinoma and higher mutational load had more chances to achieve a complete or partial response with the anti-PD-L1 antibody atezolizumab [16]. Similarly in patients with metastatic melanoma treated with CTLA-4 blockade, long-term clinical benefit was associated to the mutational burden [34]. However, mutational load alone was not sufficient to predict benefit.

Hugo et al. identified a set of transcriptomic changes related to innate anti-PD-1 resistance (IPRES signature). Pretreatment melanoma tumors from patients treated with anti-PD1 antibodies were analyzed for somatic mutanomes and transcriptomes. Twenty-one patients were classified as responders to treatment and 17 nonresponders. Twenty-six transcriptomic signatures were coenriched en bloc in 9/13 nonresponding compared with $1 / 15$ responding tumors. The signatures were associated with increased mesenchymal transition, cell adhesion inflammation, wound healing and angiogenesis identifying potential genomic determinants related to PD-1 blockades response [35]. BRCA mutations were associated with better response to the treatment.

In a different study, mutations in JAK $1 / 2$ have been associated with both intrinsic and acquired resistance to anti-PD1 antibodies. Patients with inactivating mutations of JAK $1 / 2$ are likely to have an inadequate response to IFN- $\gamma$ with low expression of PD-L1 on the tumor site and lower ability of tumor $\mathrm{T}$ cells to recognize and kill cancer cells $[36,37]$.

Despite all these biomarkers (TILs, PD-L1 expression, mutational load, etc.) have been singularly related to response to anti-PD1 blockades, none of them represent a reliable predictive factor, and none of the markers can be recommended for use in clinical practice. It is in fact more likely that all these factors are functionally interrelated and a multifactorial biomarker approach would potentially provide a stronger predictive value. Both PD-1-positive and PD-L1negative tumors can respond to treatment and PD-L1 is a dynamic marker. PD-L1 is usually assessed at baseline similarly to the other biomarkers, sometimes on archival tissue and we do not know how it changes under treatment. Longitudinal assessment of potential biomarkers would be useful to determine their variability and to evaluate potential early adaptive pathways, which could become predictive factors as well. The new studies should, therefore, include a multifactorial biomarker longitudinal assessment with the aim of identifying the better marker combination for patient selection. Selected clinical trials for theses immunotherapies are summarized in Table 1, with a timeline of their approval shown in Figure 1.

\section{Immunotherapy combinations}

In order to enhance and optimize clinical efficacy, immunotherapy can be combined with other therapeutic modalities, including chemotherapy, radiotherapy and other immunotherapy.

Chemotherapy could act in synergy with immunotherapy by promoting the release tumor antigens, the depletion of Tregs, by boosting the polarization of T-cell immunity toward TH1 


\begin{tabular}{|c|c|c|c|}
\hline Ipilimumab & $\begin{array}{l}\text { - Melanoma } \\
\text { - Melanoma }\end{array}$ & $\begin{array}{l}\text { - Ipi + gp100: } 6 \% \text { ORR; } 14 \% \text { SD; OS } 10.0 \text { vs } 6.0 \text { mo (gp100 arm) } \\
-1 \text { year OS } 36.2 \% \\
-2 \text { year OS } 17.9 \%\end{array}$ & $\begin{array}{l}{[2]} \\
{[3]}\end{array}$ \\
\hline \multirow[t]{2}{*}{ Nivolumab } & $\begin{array}{l}\text { - Melanoma } \\
\text { - Melanoma } \\
\text { - Melanoma } \\
\text { - SQ-NSCLC } \\
\text { - nonSQ-NSCLC } \\
\text { - Renal cancer }\end{array}$ & $\begin{array}{l}\text { - } 1 \text { year OS } 73 \% \text { (nivo) vs } 42 \% \text { (DTIC) } \\
\text { - ORR } 38 \% \text { (nivo) vs } 10 \% \text { (chemotherapy) } \\
\text {-2 year OS } 64 \% \text { nivolumab plus ipilimumab; } 59 \% \text { nivolumab S/A and } 45 \% \text { ipilimumab } \\
\text { - OS } 9.2 \text { mo (nivo) vs } 6.0 \text { (docetaxel) } \\
\text { - OS } 12.2 \text { mo (nivo) vs } 9.4 \text { (docetaxel) } \\
\text { - ORR } 25 \% \text { (nivo) vs } 5 \% \text { (everolimus) } \\
\text { - Median OS } 25.0 \text { mo (nivo) vs } 19.6 \text { mo (everolimus) ORR ( } 25 \text { vs } 5 \%)\end{array}$ & $\begin{array}{r}{[4]} \\
{[5]} \\
{[8]} \\
{[10]} \\
{[11]} \\
{[15]}\end{array}$ \\
\hline & - SCCHN & $\begin{array}{l}\text { - ORR } 13.3 \% \text { (nivo) vs } 5.8 \% \text { (standard therapy) } \\
\text { - Median OS } 7.5 \text { mo (nivo) vs } 5.1 \text { mo (standard therapy) }\end{array}$ & [16] \\
\hline \multirow{4}{*}{ Pembrolizumab } & $-\mathrm{NSCLC}$ & - ORR 19.4\%, PFS mo 3.7, OS 12 mo & [12] \\
\hline & $-\mathrm{NSCLC}$ & - Median PFS 10.3 mo (pembro) vs 6.0 mo (chemotherapy group) & [13] \\
\hline & - Urothelial carcinoma & - ORR $21 \%$ & [18] \\
\hline & $-\mathrm{CHL}$ & - ORR 69\%, median duration of response $11.1 \mathrm{mo}$ & [23] \\
\hline Atezolizumab & $\begin{array}{l}\text { - NSCLC } \\
\text { - Urothelial carcinoma }\end{array}$ & $\begin{array}{l}\text { - Median OS (atezo) } 13.8 \text { mo vs } 9.6 \text { mo (docetaxel) } \\
\text { - ORR } 16 \%\end{array}$ & $\begin{array}{l}{[15]} \\
{[16]}\end{array}$ \\
\hline Durvalumab & - Urothelial carcinoma & - ORR $31 \%$ & [19] \\
\hline
\end{tabular}

responses and by increasing the activation of NK cells and their number.

Furthermore, the chemotherapy itself can act as an immunomodulatory agent. It has been shown that common therapeutic agents like cyclophosphamide and gemcitabine when given at low doses or metronomically can modulate the tumor microenvironment favoring the immune response [38]. Low doses of MTX can enhance antigen processing by dendritic cells (DCs), and doxorubicin and paclitaxel are able to upregulate HLA and enhance DC functions.

Clinical data about combination strategies of immunotherapy with chemotherapy are limited. The chemotherapy effect of releasing tumor antigens is likely, however, to play an important role as patients with tumors traditionally chemoresponsive, like NSCLC, seem to gain more benefit compared with cancer chemoresistant, like melanoma. Despite the lack of a direct comparison, the outcomes for melanoma patients seen with combination ipilimumab plus dacarbazine are very similar to the outcomes seen with single agent ipilimumab [3]. Results were more promising when anti-PD1 antibodies were combined with chemotherapy in metastatic gastric cancer and NSCLC and the combination is currently under investigation in these two cancer entities in large Phase III trials.

Targeted agents can synergize with immunotherapy by increasing tumor antigenicity, by promoting T-cell migration into the tumor and by increasing PD-L1 expression on the tumor, as seen for BRAFi. MAPK inhibitors, can complement T-cell checkpoint therapies by enhancing tumor antigen expression, immunogenic tumor cell death and T-cell infiltration into tumors. VEGF inhibitors can complement T-cell checkpoint therapies by enhancing DC maturation and activity as well as T-cell infiltration into tumor [39]. A possible disadvantage is the inhibition of pathways involved in the immune response, but data regarding this issue are still controversial. Improved antitumor activity has been observed with the combination of immunotherapy with BRAF and MEK inhibitors in BRAFV600E melanoma, but MEK inhibitors also have been shown to have negative 


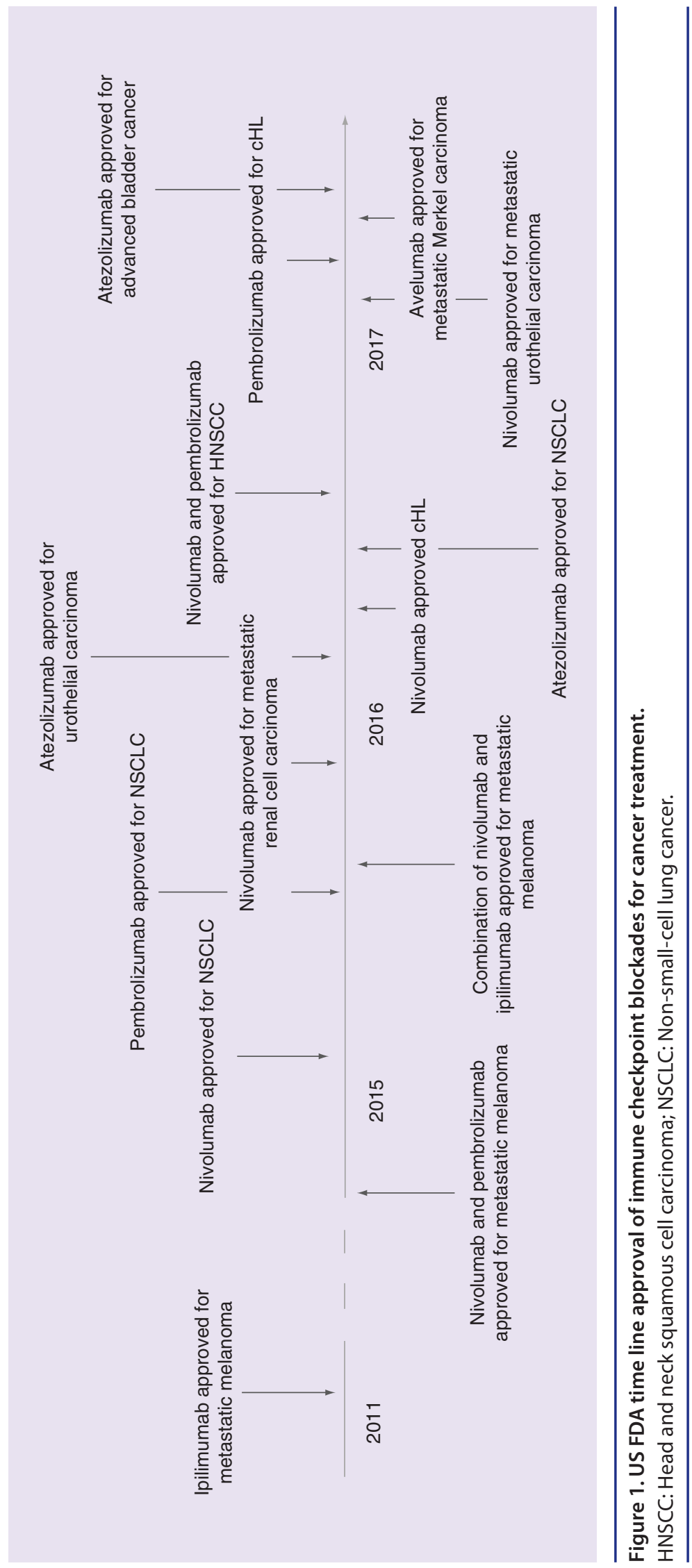

effects on naive T-cell proliferation, viability and IFN- $\gamma$ secretion [39]. However, in clinical practice a major problem of such a combination is the tolerability. The Phase I study combining ipilimumab and vemurafenib had to be stopped due to the high liver toxicity despite a strong rationale of using this combination. The BRAFi vemurafenib has shown in fact to enhance T-cell recognition of melanoma [40] improves antitumor activity of adoptive cell immunotherapy [41], and it increases tumor infiltration by $\mathrm{T}$ cells [42] Further combinations have been considered and some of them are currently under investigation in Phase III trials with a more acceptable toxicity profile. The combination of dabrafenib with ipilimumab, for instance, appeared to be tolerable and has not been associated with significant hepatotoxicity in melanoma. On the contrary, the combination of dabrafenib, trametinib with ipilimumab was stopped early after two out of seven patients developed colon perforation soon after initiating ipilimumab therapy.

Immunotherapy can be combined with radiotherapy. Radiotherapy can promote release of tumor antigen, inflammation and migration of cells of the immune system to the irradiated tumor (cold to hot tumor), can increase the number of neoantigens making the tumor more recognizable by the immune system and can promote the activation of immunogenic death pathways. But the most interesting effect seen with such a combination is the so-called abscopal effect (out-of-target tumor response). The best example of this is the study of Kolstad et al., which showed that combining radiotherapy with intranodal injection of low-dose rituximab, immature DCs and granulocyte-macrophage colonystimulating factor (GM-CSF) in patients with disseminated follicular lymphoma can induce systemic CD8 T-cell immunity and tumor regression [43].

The combination of two different immunotherapeutics has many potential advantages. Different agents can in fact boost the immune response by acting on different levels and on different stages of the immune response. By acting predominantly on early or late stages of the immune response we can modulate the specificity of the response, being less specific or more specific, respectively. By acting on the afferent phase of the immune response we can increase the magnitude of T-cell response and their avidity. By predominantly acting on the efferent phase we would be able to increase the 
trafficking and to modulate tolerance mechanism. Anti-CTLA4 and anti-PD1 therapies are synergistic when combined together because of their complementary effects on T-cell priming in the lymph nodes and T-cell activation and killing in the tumors. We have previously discussed the findings of the CheckMate 067 study, which evaluated the combination ipilimumab and nivolumab against single agent treatment, but we did not mention the toxicity profile. This is one of the challenges for the clinicians as the combination showed poor tolerability as more than $50 \%$ of the patients experienced grade 3 and 4 toxicities. The most of these toxicities were immune related and some of them are chronic. New combination strategies are warranted in order to reduce toxicity. The CheckMate study 067 is a Phase II study, which evaluated the combination of ipilimumab and nivolumab given sequentially rather than concurrently with a planned switch. The treatment given sequentially did not show a better toxicity profile. Furthermore, the cohort of patients receiving ipilimumab first, followed by nivolumab, did unexpectedly significantly worse in terms of ORR and progression rate compared with the cohort of patients receiving nivolumab first.

The combination of these agents with TILs or modified $T$ cells can help to address this issue as driving a more specific tumor immune response might improve the toxicity profile, avoiding the immune-related adverse events we see with the checkpoint blockades. Preliminary results are encouraging, but it is still too early to see such a kind of combination in late phase trials.

\section{Novel immunotherapeutics \& future perspective}

It is important to realize just how many molecules can be included under the title of 'Checkpoint.' CTLA-4 and PD-1 are examples of inhibitors that include many others, including TIM-3, BTLA, VISTA and LAG-3. Checkpoints can also be stimulated with agonist antibodies such as CD28, 0X40, GITR, CD137, CD27 and HVEM. Among these, the novel anti-CD137, urelumab, has already entered Phase II trials. Urelumab demonstrated antitumor activity through the enhancement of T cells and NK activities. In a Phase I-II combination study with the nivolumab, 33 out of 71 patients had a reduction in the tumor burden to RECIST criteria. Combinations of an agonist and antagonist are attractive in theory but need to be tempered by the potential side effects exemplified by the Northwick Park Phase I disaster with a CD28-based antibody. Nevertheless, CD27 (varlilumab) is being developed with an anti-PD-L1 antibody (atezolizumab) by Celldex (NJ, USA). Combinations currently in development, interestingly, include many candidates that have failed to be effective in previous studies, such as tumor antigen vaccines, as well as agents with marked activity in a small percentage of patients, for example, intratumoral agents such as talimogene laherparepvec (T-VEC). Idera Pharmaceuticals (MA, USA) are using an intratumoral TLR9 agonist to modulate the tumor microenvironment to enhance antitumor immunity (together with ipilimumab). Other approaches include combining with anti-KIR (lirilumab) to target NK cells with both ipilimumab and nivolumab. To date, all checkpoints have been intravenous antibodies, but this may change as Curis (MA, USA) has announced that they have an oral checkpoint inhibitor that is very encouraging in preclinical trials (CA170).

Beyond the checkpoints, many high-tech approaches are being pursued, such as antitumor vaccination, oncolytic strategies, bispecific antibodies, CAR T cells, etc. However, we may have overlooked very basic low tech approaches in the rush to develop more and more sophisticated agents. These include simple immune modulators/anti-inflammatories, such as the IMiDs, IMM-101, many drugs in low doses, such as cyclophosphamide and gemcitabine and the use of basic TLR agonists and already developed cytokines. The most impressive studies have used lenalidomide and pomalidomide, which, in addition to inhibiting COX-2, are very costimulatory [44]. It is a logical step to prime with these agents in any immunotherapy strategy.

This may or may not be linked to the role of activated mononuclear phagocytes/macrophages in the outcome of checkpoint therapy [45] where it has been shown that the induction of the $\mathrm{CD}_{103^{+}}$marker on DCs is an absolute required for PD-1-based therapy to be effective in a murine model.

This is of interest as a review of many different vaccine studies over the last two decades highlighted the excellent long-term outcome of patients given a heat-killed mycobacterium vaccine preparation (also known as SRL-172) with stage IV melanoma patients achieving similar 5- and 8-year survivals to that reported using ipilimumab. Unfortunately, by the time this was 
evident SR Pharma (Halifax, UK) had dropped the project to pursue more high-tech approaches. Further research suggested that a closely related agent (Mycobacterium obuense) was as effective and much easier to produce to good manufacturing practice and Immodulon (London, UK) have now produced this as IMM-101, which has been shown to be active alone in melanoma [46] and with gemcitabine versus gemcitabine alone in a randomized study in advanced pancreatic cancer patients [47]. This agent has the advantages of being a TLR-2 and 4 agonist, having multiple antigens, many shared with tumors, as well as the ability to activate both innate (NK and $\gamma \delta$ $\mathrm{T}$ cells) and the cell mediated (Th-1) response. In addition, it can activate macrophages. More importantly, it has no added side effects, being a very small intradermal $(0.1 \mathrm{ml})$ injection with no negative systemic effects at all.

This agent has already been shown to increase the effects of low dose IL-2, gemcitabine, zoledronic acid and carboplatin-based chemotherapy, as well as radiotherapy. Early anecdotal data (reported at SITC 2016) suggest that patients pretreated with IMM-101, who progress, have had exceptional responses to ipilimumab and pembrolizumab and a combined study has now commenced to explore this potential synergy.

A combinatorial immunotherapeutic strategy of combining CAR T cells with checkpoint blockade is a promising treatment approach for solid tumors. In a murine model of mesothelioma, Cherkassky et al. [48] showed that high doses of CD28 and 4-1BB-based CAR T cells were able to eradicate the tumor. 4-1BB CAR T cells retained their cytotoxic and cytokine secretion functions longer than CD28 CAR T cells, and their prolonged function correlated with improved survival. Only when a PD-1-blocking antibody was added, the effector function of exhausted CD28 CAR T cells was restored suggesting that PD1 blockade may overcome CAR T-cell exhaustion often observed in solid tumors.

Oncolytic viruses are another very promising strategy and a wide variety of oncolytic viruses are currently under clinical development worldwide. They consist of genetically engineered or naturally occurring viruses that selectively replicate in and kill cancer cells. In a Phase III clinical trial, T-VEC showed to improve the outcome of patients with metastatic melanoma. T-VEC is based on herpes simplex virus type 1 and has been modified to include a gene that codes for GM-CSF. In the study for T-VEC showed an ORR of 26.4 versus
$5.7 \%$ of the control arm consisting of subcutaneous administration of GM-CSF with median OS of 23.3 months with T-VEC versus 18.9 months with GM-CSF [49]. Oncolytic viruses can be combined with immune checkpoint blockades as currently under investigation for T-VEC or with nonimmunotherapeutical agents. Lawson et al. [50] have evaluated the combination of sunitinib with a reovirus in a murine model of renal cancer and observed that the combination of sunitinib with reovirus was able to improve survival and to promote the development of protective immunity upon tumor rechallenge compared with sunitinib single agent or reovirus alone.

Bispecific antibodies are capable of binding two targets simultaneously. They are produced as recombinant proteins with two antigen-binding regions with or without Fc. Bispecific antibodies can be used to block two different signaling pathways simultaneously, to dual target different disease mediators or to simply recruit cells of the immune system to the tumor site. Catumaxomab is an anti-EpCAM-anti-CD3 antibody approved in the EU for the treatment of recurrent malignant ascites [51], and blinatumomab is an anti-CD19 and anti-CD3 antibody approved in the USA for the treatment of Philadelphia chromosome-negative relapsed or refractory acute lymphoblastic leukemia [52]. Bispecific antibodies might have a role in combination with checkpoint blockades. Presence of TILs and tumor-specific T cells correlate with response to anti-PD1 antibodies. Recruitment of T cells at tumor level, retargeting of $\mathrm{T}$ cells to tumor and activation of $\mathrm{T}$ cells may, therefore, increase the activity of immune checkpoint blockades and switch nonresponders into responders.

Another very interesting approach consist of the so-called nanovaccines. In a preclinical model of Kim et al. showed that nanoparticles encapsulating a imidazoquinoline derivative that activates both toll-like receptor 7 and 8 used in combination with a model antigen were able to improve DC activation in the lymph node, increase the activation of $\mathrm{T}$ cells and of frequency of antigen-specific $T$ cells [53].

One last point is whether the fixation on combinations can be overdone when it may be more important to get the sequence right and this is not always predictable on known scientific data, for example, the poor results with nivolumab given after ipilimumab, compared with the other way round was the complete opposite of what was predicted, highlighting 


\section{EXECUTIVE SUMMARY}

- In 2016, cancer immunotherapy has been named Advance of the Year by the American Society of Clinical Oncology.

\section{Checkpoint blockades: state of art}

- The CTLA-4 antibody ipilimumab and the anti-PD1 antibodies pembrolizumab and nivolumab have granted approval for the treatment of metastatic melanoma, and they are the new standard of care for this disease. Nivolumab and pebmbrolizumab received approval for the treatment of patients with non-small-cell lung cancer whose disease has progressed after at least one line of treatment. Atezolizumab was the first ever anti-PD-L1 antibody approved by US FDA. It is indicated for patients with metastatic non-small-cell lung cancer whose disease progressed during or following platinum-containing chemotherapy. Significant efficacy and prolongation of progression-free survival and overall survival has been observed for nivolumab also in patients with metastatic renal cancer and advanced head and neck cancer. Nivolumab has received approval for the treatment of renal cancer patients who have received prior antiangiogenic therapy and for the treatment of patients with recurrent or metastatic squamous cell carcinoma of the head and neck with disease progression on or after platinum-based therapy. Pembrolizumab and nivolumab are approved treatment for refractory or recurrent classical Hodgkin lymphoma based on Phase Il studies results. Continued approval for this indication will depend on the outcome of ongoing randomized Phase III studies.

Predictive biomarkers, more than just PD-1/PDL-1

- Studies suggested that while PD-L1 expression has potential as a predictive biomarker, it cannot be currently used for patient selection for single agent anti-PD1s as a significant number of patients classified as PD-L1 negative still benefit from treatment.

- Emerging data have suggested that biomarkers based on immunoprofiling and mismatch repair deficiency may be more useful predictors of treatment response than PD-L1.

- A multifactorial biomarker approach can potentially provide a stronger predictive value.

\section{Immunotherapy combinations}

- In order to enhance and optimize clinical efficacy, immunotherapy can be combined with other therapeutic modalities including chemotherapy, radiotherapy and other immunotherapy.

- Chemotherapy and targeted agents could act in synergy with immunotherapy by promoting the release tumor antigens, the depletion of Tregs, by boosting the polarization of T-cell immunity toward TH1 responses and by increasing the activation of natural killer cells and their number. Furthermore, BRAFi can potentially increase PD-L1 expression.

- Radiotherapy can synergize with immunotherapy by increasing the number of neoantigens making the tumor more recognizable by the immune system and by promoting the activation of immunogenic death pathways.

- Combination of checkpoint inhibitors is a promising strategy; however, it is limited by the increased toxicity.

Novel immunotherapeutics \& future perspective

- New checkpoint inhibitors include anti- TIM-3, BTLA, VISTA and LAG-3. Checkpoints can also be stimulated with agonist antibodies such as CD28, 0X40, GITR, CD137, CD27 and HVEM.

- Beyond the checkpoints, many high-tech approaches are being pursued, such as oncolytic intratumoral strategies (talimogene laherparepvec), bispecific antibodies, CAR T cells, etc., but except for talimogene laherparepvec none has entered clinical practice so far.

\section{Conclusion}

- Different approaches may have a different potential in gaining benefit for different patients. Treatment with checkpoint blockades should be optimized in order to increase benefit minimizing the toxicity.

- A potential strategy is to establish a baseline 'immunogenicity score' using a panel of biomarkers which includes microenvironment-related biomarkers and select the most appropriate strategy (more aggressive versus less aggressive) based on this score.

- This would require a deeper understanding of the biology of the tumor in the context of immunotherapy, including the understating of the mechanisms of acquired resistance to immunotherapy. 
the importance of performing trials with several permutations [54].

\section{Conclusion}

Most healthcare systems are cracking at the seams with the unexpected cost of new cancer agents, especially when they often give only an extra survival of a few months in many cases. Combinations of such expensive drugs, which can cost up to $£ 100 \mathrm{~K}$ a year is seen as a step too far by even those involved in their development. This means that there must be a place for back to basic, simpler approaches, which might just prime the tumor environment to respond better to checkpoint inhibitors.

Previous immunotherapy has been focused on cytokines (interferons and interleukins), TLR agonists, such as TLR7 and TLR9, and vaccination-based strategies. Cytokines, such as IL-2 expand activated T cells even at nontoxic low levels and should not be overlooked in optimizing checkpoint inhibitor strategies.

Different approaches may have a different potential in gaining benefit for different patients. We would need a strategy for personalized cancer immunotherapy to increase the therapeutic index. A potential strategy is to establish a baseline 'immunogenicity score' using a panel of biomarkers that include microenvironment-related biomarkers [55]. Tumors with high score and a responsive microenvironment may be treated with monotherapy targeting $\mathrm{T}$-cell response. Tumors with a low score would need a more aggressive approach targeting both $\mathrm{T}$ cells and microenvironment.

However, this would require a deeper understanding of the biology of the tumor in the context of immunotherapy, including the understating of the mechanisms of acquired resistance to immunotherapy. A new challenge that we are facing now as we have started seeing patients becoming resistant to treatment even after a long period of benefit.

\section{Financial \& competing interests disclosure}

The authors have no relevant affiliations or financial involvement with any organization or entity with a financial interest in or financial conflict with the subject matter or materials discussed in the manuscript. This includes employment, consultancies, honoraria, stock ownership or options, expert testimony, grants or patents received or pending, or royalties.

No writing assistance was utilized in the production of this manuscript

\section{Open access}

This work is licensed under the AttributionNonCommercial-NoDerivatives 4.0 Unported License. To view a copy of this license, visit http://creativecommons.org/ licenses/by-nc-nd/4.0/

\section{References}

1 Kirkwood JM, Tarhini AA, Panelli MC et al. Next generation of immunotherapy for melanoma. J. Clin. Oncol. 26(20), 3445-3455 (2008).

2 Hodi FS, O’Day SJ, Mcdermott DF et al. Improved survival with ipilimumab in patients with metastatic melanoma. N. Engl. J. Med. 363(8), 711-723 (2010).

3 Robert C, Thomas L, Bondarenko I et al. Ipilimumab plus dacarbazine for previously untreated metastatic melanoma. $N$. Engl. J. Med. 364(26), 2517-2526 (2011).

4 Schadendorf D, Hodi FS, Robert C et al. Pooled analysis of long-term survival data from Phase II and Phase III trials of ipilimumab in unresectable or metastatic melanoma. J. Clin. Oncol. 33(17), 1889-1894 (2015).

5 Robert C, Long GV, Brady B et al. Nivolumab in previously untreated melanoma without BRAF mutation. N. Engl. J. Med. 372(4), 320-330 (2015).
6 Weber JS, D’angelo SP, Minor D et al. Nivolumab versus chemotherapy in patients with advanced melanoma who progressed after anti-CTLA-4 treatment (CheckMate 037): a randomised, controlled, open-label, Phase III trial. Lancet Oncol. 16(4), 375-384 (2015).

7 Larkin J, Chiarion-Sileni V, Gonzalez R et al. Combined nivolumab and ipilimumab or monotherapy in untreated melanoma. N. Engl. J. Med. 373(1), 23-34 (2015).

8 Larkin J, Chiarion-Sileni V, Gonzalez R et al. CT075 - Overall survival (OS) results from a Phase III trial of nivolumab (NIVO) combined with ipilimumab (IPI) in treatment-naïve patients with advanced melanoma (CheckMate 067). Presented at: AACR Annual Meeting 2017. DC, USA. 1-5 April 2017.

9 Daud AI, Wolchok JD, Robert C et al. Programmed death-ligand 1 expression and response to the anti-programmed death 1 antibody pembrolizumab in melanoma. J. Clin. Oncol. 34(34), 4102-4109 (2016).
10 Robert C, Karaszewska B, Schachter J et al. Improved overall survival in melanoma with combined dabrafenib and trametinib. N. Engl. J. Med. 372(1), 30-39 (2015).

11 Brahmer J, Reckamp KL, Baas P et al. Nivolumab versus docetaxel in advanced squamous-cell non-small-cell lung cancer. N. Engl. J. Med. 373(2), 123-135 (2015).

12 Borghaei H, Paz-Ares L, Horn L et al. Nivolumab versus docetaxel in advanced nonsquamous non-small-cell lung cancer. N. Engl. J. Med. 373(17), 1627-1639 (2015).

13 Garon EB, Rizvi NA, Hui R et al. Pembrolizumab for the treatment of non-small-cell lung cancer. N. Engl. J. Med. 372(21), 2018-2028 (2015).

14 Reck M, Rodriguez-Abreu D, Robinson AG et al. Pembrolizumab versus chemotherapy for PD-L1-positive non-small-cell lung cancer. N. Engl. J. Med. 375(19), 1823-1833 (2016).

15 Rittmeyer A, Barlesi F, Waterkamp D et al. Atezolizumab versus docetaxel in patients 
with previously treated non-small-cell lung cancer (OAK): a Phase III, open-label, multicentre randomised controlled trial. Lancet 389(10066), 255-265 (2017).

16 Rosenberg JE, Hoffman-Censits J, Powles T et al. Atezolizumab in patients with locally advanced and metastatic urothelial carcinoma who have progressed following treatment with platinum-based chemotherapy: a single-arm, multicentre, Phase II trial. Lancet 387(10031), 1909-1920 (2016).

17 Sharma P, Callahan MK, Bono P et al. Nivolumab monotherapy in recurrent metastatic urothelial carcinoma (CheckMate 032): a multicentre, open-label, two-stage, multi-arm, Phase I/II trial. Lancet Oncol. 17(11), 1590-1598 (2016).

18 Bellmunt J, de Wit R, Vaughn DJ et al. Pembrolizumab as second-line therapy for advanced urothelial carcinoma. N. Engl. J. Med. 376(11), 1015-1026 (2017).

19 Massard C, Gordon MS, Sharma S et al. Safety and efficacy of durvalumab (MEDI4736), an anti-programmed cell death ligand-1 immune checkpoint inhibitor, in patients with advanced urothelial bladder cancer. J. Clin. Oncol. 34(26), 3119-3125 (2016).

20 Motzer RJ, Escudier B, Mcdermott DF et al. Nivolumab versus everolimus in advanced renal-cell carcinoma. N. Engl. J. Med. 373(19), 1803-1813 (2015).

21 Ferris RL, Blumenschein G Jr, Fayette J et al. Nivolumab for recurrent squamous-cell carcinoma of the head and neck. N. Engl. J. Med. 375(19), 1856-1867 (2016).

22 Younes A, Santoro A, Shipp M et al. Nivolumab for classical Hodgkin's lymphoma after failure of both autologous stem-cell transplantation and brentuximab vedotin: a multicentre, multicohort, single-arm Phase II trial. Lancet Oncol. 17(9), 1283-1294 (2016).

23 Chen RW, Zinzani PL, Fanale MA et al. Pembrolizumab for relapsed/refractory classical Hodgkin lymphoma (R/R cHL): Phase II KEYNOTE-087 study. J. Clin. Oncol. 34(Suppl.), Abstract 7555 (2016).

24 Robert C, Schachter J, Long GV et al. Pembrolizumab versus ipilimumab in advanced melanoma. $N$. Engl. J. Med. 372(26), 2521-2532 (2015)

25 Tumeh PC, Harview CL, Yearley JH et al. PD-1 blockade induces responses by inhibiting adaptive immune resistance. Nature 515(7528), 568-571 (2014).

26 Ji RR, Chasalow SD, Wang L et al. An immune-active tumor microenvironment favors clinical response to ipilimumab. Cancer
Immunol. Immunother. 61(7), 1019-1031 (2012).

27 Spranger S, Bao R, Gajewski TF. Melanomaintrinsic beta-catenin signalling prevents anti-tumour immunity. Nature 523(7559), 231-235 (2015).

28 Le DT, Uram JN, Wang H et al. PD-1 blockade in tumors with mismatch-repair deficiency. N. Engl. J. Med. 372(26), 2509-2520 (2015).

29 Weber J, Roder H, Asmellash S et al. Abstract 4891: a mass spectrometry-based serum test to predict outcome of treatment with nivolumab: analysis of samples taken during therapy. Cancer Res. 76(Suppl. 14), Abstract nr 4891 (2016).

30 Dalgleish AG, O’Byrne KJ. Chronic immune activation and inflammation in the pathogenesis of AIDS and cancer. Adv. Cancer Res. 84, 231-276 (2002).

31 Chan TA, Riaz N, Havel JJ et al. Immunogenomic analyses of tumor cells and microenvironment in patients with advanced melanoma before and after treatment with nivolumab. Presented at: AACR Annual Meeting 2017. DC, USA. 1-5 April 2017.

32 Lawrence MS, Stojanov P, Polak P et al. Mutational heterogeneity in cancer and the search for new cancer-associated genes. Nature 499(7457), 214-218 (2013).

33 Rizvi NA, Hellmann MD, Snyder A et al. Cancer immunology. Mutational landscape determines sensitivity to PD-1 blockade in non-small cell lung cancer. Science 348(6230), 124-128 (2015).

34 Snyder A, Makarov V, Merghoub Tet al. Genetic basis for clinical response to CTLA-4 blockade in melanoma. N. Engl. J. Med. 371(23), 2189-2199 (2014).

35 Hugo W, Zaretsky JM, Sun L et al. Genomic and transcriptomic features of response to anti-PD-1 therapy in metastatic melanoma. Cell 165(1), 35-44 (2016).

36 Shin DS, Zaretsky JM, Escuin-Ordinas H et al. Primary resistance to PD-1 blockade mediated by JAK1/2 mutations. Cancer Discov. 7(2), 188-201 (2017).

37 Zaretsky JM, Garcia-Diaz A, Shin DS et al. Mutations associated with acquired resistance to PD-1 blockade in melanoma. $N$. Engl. J. Med. 375(9), 819-829 (2016).

38 Fryer RA, Barlett B, Galustian C, Dalgleish AG. Mechanisms underlying gemcitabine resistance in pancreatic cancer and sensitisation by the $\mathrm{iMiD}^{\mathrm{TM}}$ lenalidomide. Anticancer Res. 31(11), 3747-3756 (2011).

39 Hughes PE, Caenepeel S, Wu LC. Targeted therapy and checkpoint immunotherapy combinations for the treatment of cancer. Trends Immunol. 37(7), 462-476 (2016).

40 Boni A, Cogdill AP, Dang P et al. Selective BRAFV600E inhibition enhances T-cell recognition of melanoma without affecting lymphocyte function. Cancer Res. 70(13), 5213-5219 (2010).

41 Koya RC, Mok S, Otte N et al. BRAF inhibitor vemurafenib improves the antitumor activity of adoptive cell immunotherapy. Cancer Res. 72(16), 3928-3937 (2012).

42 Liu C, Peng W, Xu C et al. BRAF inhibition increases tumor infiltration by $\mathrm{T}$ cells and enhances the antitumor activity of adoptive immunotherapy in mice. Clin. Cancer Res. 19(2), 393-403 (2013).

43 Kolstad A, Kumari S, Walczak M et al. Sequential intranodal immunotherapy induces antitumor immunity and correlated regression of disseminated follicular lymphoma. Blood 125(1), 82-89 (2015).

44 Dredge K, Marriott JB, Todryk SM et al. Protective antitumor immunity induced by a costimulatory thalidomide analog in conjunction with whole tumor cell vaccination is mediated by increased Th1-type immunity. J. Immunol. 168(10), 4914-4919 (2002).

45 Salmon H, Idoyaga J, Rahman A et al. Expansion and activation of CD103(+) dendritic cell progenitors at the tumor site enhances tumor responses to therapeutic PD-L1 and BRAF inhibition. Immunity 44(4), 924-938 (2016).

46 Stebbing J, Dalgleish A, Gifford-Moore A et al. An intra-patient placebo-controlled Phase I trial to evaluate the safety and tolerability of intradermal IMM-101 in melanoma. Ann. Oncol. 23(5), 1314-1319 (2012).

47 Dalgleish AG, Stebbing J, Adamson DJ et al. Randomised, open-label, Phase II study of gemcitabine with and without IMM-101 for advanced pancreatic cancer. Br. J. Cancer 115(9), e16 (2016).

48 Cherkassky L, Morello A, Villena-Vargas J et al. Human CAR T cells with cell-intrinsic PD-1 checkpoint blockade resist tumormediated inhibition. J. Clin. Invest. 126(8), 3130-3144 (2016).

49 Andtbacka RH, Kaufman HL, Collichio F et al. Talimogene laherparepvec improves durable response rate in patients with advanced melanoma. J. Clin. Oncol. 33(25), 2780-2788 (2015).

50 Lawson KA, Mostafa AA, Shi ZQ et al. Repurposing sunitinib with oncolytic reovirus as a novel immunotherapeutic strategy for 
REVIEW Fusi \& Dalgleish

renal cell carcinoma. Clin. Cancer Res. 22(23), 5839-5850 (2016).

51 Seimetz D, Lindhofer H, Bokemeyer C. Development and approval of the trifunctional antibody catumaxomab (anti-EpCAM x anti-CD3) as a targeted cancer immunotherapy. Cancer Treat. Rev. 36(6), 458-467 (2010).

52 Martinelli G, Boissel N, Chevallier P et al. Complete hematologic and molecular response in adult patients with relapsed/ refractory Philadelphia chromosome-positive B-precursor acute lymphoblastic leukemia following treatment with blinatumomab: results from a Phase II, single-arm, multicenter study. J. Clin. Oncol. doi:10.1200/JCO.2016.69.3531 (2017) (Epub ahead of print).

53 Hyunjoon K, L Niu, Larson P et al. Nanovaccine: a novel immunotherapeutic strategy to treat bladder cancer (abstract). Cancer Res. 76(Suppl. 14), Abstract 2348 (2016).
54 Weber JS, Gibney G, Sullivan RJ et al. Sequential administration of nivolumab and ipilimumab with a planned switch in patients with advanced melanoma (CheckMate 064): an open-label, randomised, Phase II trial. Lancet Oncol. 17(7), 943-955 (2016).

55 Sathyanarayanan V, Neelapu SS. Cancer immunotherapy: strategies for personalization and combinatorial approaches. Mol. Oncol. 9(10), 2043-2053 (2015). 